\title{
Electromagnetic Navigation Bronchoscopy
}

National Cancer Institute

\section{Source}

National Cancer Institute. Electromagnetic Navigation Bronchoscopy. NCI Thesaurus. Code C116479.

A two-stage bronchoscopic technique that uses electromagnetic technology to first prepare 3D, digital reconstructions of the patient's airways, and then helps the clinician use those images to guide instruments through the bronchi to the previously identified target. This is most often performed to biopsy lesions or lymph nodes, or place a brachytherapy catheter. 